\title{
Generalized Chorea Induced by an Unilateral Anterior Cerebral Artery Territorial Infarction
}

\section{Jae Hong Chang \\ Woo-Keun Seo \\ Moon-Ho Park \\ Jong-Mun Lee \\ Do-Young Kwon \\ Seong-Beom Koh}

Departments of Neurology

College of Medicine,

Korea University, Seoul, Korea
Received May 7, 2008

Revised June 13, 2008 Accepted July 21, 2008

\section{Corresponding author}

Woo-Keun Seo, MD, PhD

Department of Neurology,

College of Medicine,

Korea University,

80 Guro-dong, Guro-gu,

Seoul 152-703, Korea

Tel $+82-2-2626-3172$

Fax $+82-2-2626-1257$

E-mail nukseo@korea.ac.kr
Generalized chorea caused by unilateral cerebral infarction has rarely been reported. A 58 -yearold woman presented involuntary movement in her all extremities after acute cerebral infarction on her right anterior cerebral artery territory. The involuntary movements were diagnosed as generalized chorea. We didn't find any cause of generalized chorea except the acute cerebral infarction. Here, we described the case of generalized chorea after unilateral cerebral infarction discussing the possible mechanisms. Journal of Movement Disorders 2009;2:37-39

Key Words: Chorea, Brain infarction, Anterior cerebral artery.

\section{Introduction}

Chorea or hyperkinetic movement disorders caused by stroke were reported in $1 \%$ of patient with acute stroke. ${ }^{1}$ However, generalized chorea caused by unilateral lesion has rarely been reported. Here, we report a case of generalized chorea after acute cerebral infarction in unilateral anterior cerebral artery (ACA) territory discussing the possible mechanisms.

\section{Case Report}

A 58-year-old woman was admitted presenting with involuntary movement in her all extremities for 1 day. She denied any history of medication, involuntary movement, preceding infection or family history. On admission, blood pressure was 190/100 mmHg and a general physical examination was normal. She was alert and fully awaked. Neuropsychologic tests revealed ideomotor apraxia and alien hand syndrome presented with compulsive manipulation of tools or reflexive grasping in her left side. The examination for cranial nerves, sensory and deep tendon reflexes were normal except a subtle dysarthria and left leg weakness. Chorea was observed in her distal parts of four limbs with non-rhythmic and rotatory in nature. The choreic movements attenuated in a relaxed state, and disappeared during sleep.

Laboratory data were unremarkable with normal fasting serum glucose level (119 mg/dL), white blood cell count $(7,600 / \mu \mathrm{L})$, hemoglobin $(13.2 \mathrm{~g} / \mathrm{dL})$, peripheral blood morphology, liver enzymes, and thyroid function tests. Electroencephalogram was normal. Brain magnetic resonance images revealed acute ischemic stroke involving the right cingulate gyrus, genu and anterior body of corpus callosum and an old infarction on left caudate nucleus was seen (Figure 1). Atrophy of caudate nucleus was not seen. CT angiography revealed obstruction of right ACA after anterior communicating artery and mild stenosis of left middle cerebral artery. On perfusion CT scan, decreased cerebral blood volume and cerebral blood flow and delayed mean transit time and time to peak were observed on the ischemic stroke lesion and no perfusion defect were found on basal ganglia, thalamus, and subthalamic area. Chorea diminished after trying haloperidol. Chorea of right side improved earlier than that of left side. She was discharged with subtle chorea in her left side on ninth hospital day. At six months from onset of symptoms, without haloperidol medication, dysarthria and weakness of her left leg disappeared and only subtle chorea on her distal limbs remained. 


\section{Discussion}

Generalized chorea caused by stroke is uncommon condition. In a series of post-stroke chorea, 2 cases among 20 cases had generalized chorea. ${ }^{2}$ However the 2 cases with generalized chorea had bilateral lesions on thalamus or cortex. No
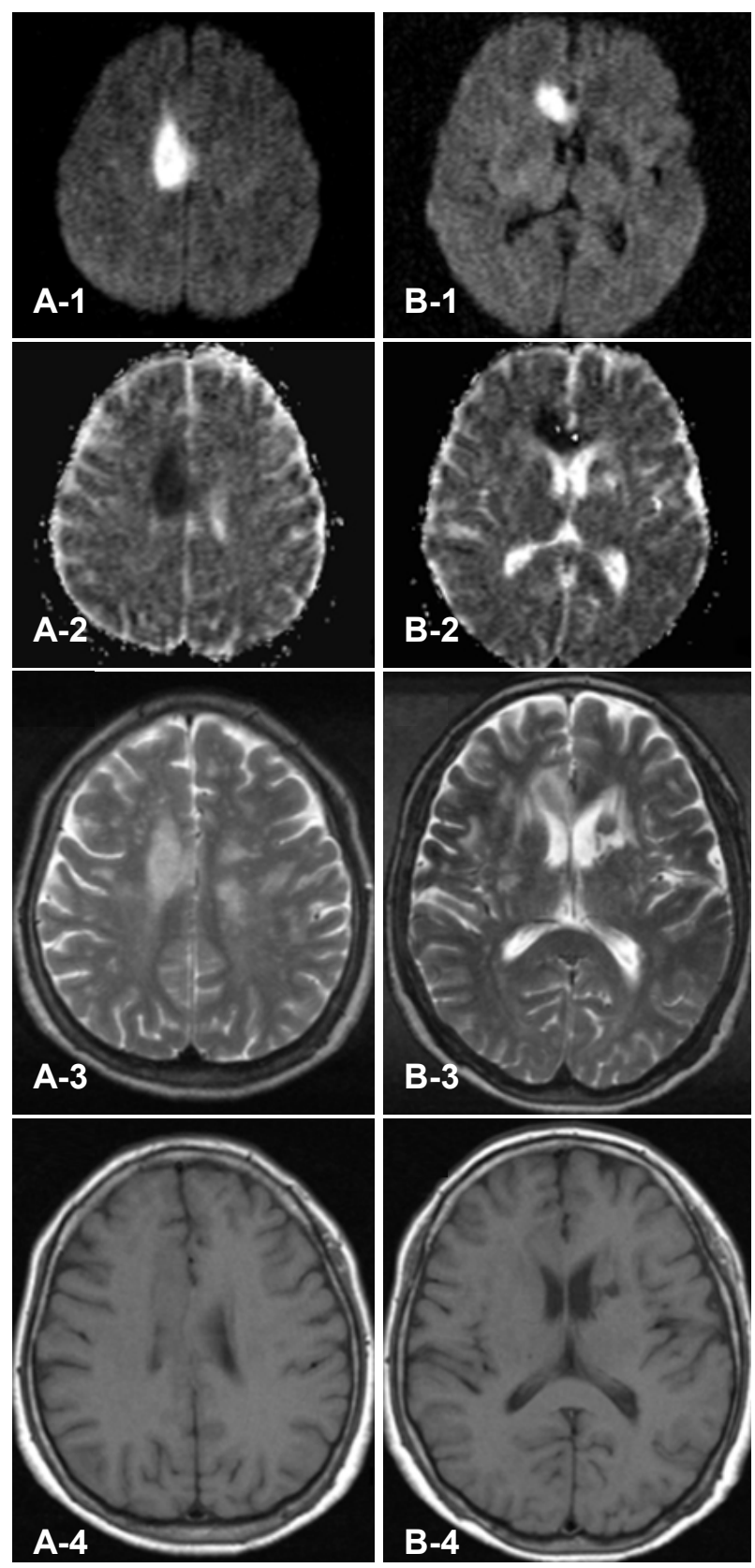

Figure 1. Magnetic resonance images at the level of right cingulate gyrus (A1-A4) and corpus callosum (B1-B4). The lesions had high signal intensities on diffusion weighted images (A1, B1) and low signal intensities on apparent diffusion coefficient map (A2, B2). T2-weighted images showed high signal intensity on right cingulate gyrus, corpus callosum, and left caudate nucleus (A3, B3). T1weighted images showed low signal intensity lesion on left caudate nucleus (B4) and isosignal or subtle low signal intensities on the right cingulate gyrus (A4) and corpus callosum (B4). specific cause of chorea was found in the presented case such as family history of neurologic disease, personality changes, medication, and abnormal laboratory findings. Although genetic studies for the diseases that could cause generalized chorea were not performed, the spontaneous resolution of chorea without a recurrence suggested that the possibility of genetic cause was very low. Our case had acute ischemic stroke that was limited in right side and no perfusion defect was found on the contralateral side. The choreic movements developed acutely accompanying with acute focal neurological deficits which could be explained by acute cerebral infarction. Therefore, we considered that the acute ischemic stroke in this patient was responsible for generalized chorea.

The pathogenesis of hyperkinetic involuntary movements such as chorea or ballism has been recognized as disinhibition and functional disconnection in thalamocorticobasal ganglionic circuitry. ${ }^{2,3}$ Lesions of STN, striatum or the pars externa of the globus pallidus reduce excitatory projection from STN to the pars interna of the globus pallidus, which in turn disinhibit the thalamus and cortex, resulting in hyperkinetic movements. ${ }^{3}$ Supplementary motor area (SMA) also has anatomical connections with the striatum and functional disconnection between the striatum and SMA can produce involuntary movement. ${ }^{5,6}$ Therefore, ACA territorial infarction could produce contralateral hemichorea. $\mathrm{Kim}^{7}$ reported that 9 patients with ACA infarction had abnormal involuntary movement syndrome such as parkinsonism or asterixis. However among the patients, chorea was not found and 1 patient showed bilateral asterixis which was severe on contralateral side of lesion.

Ipsilateral hemichorea by stroke has been rarely reported and the pathophysiology is still less understood. ${ }^{8-10}$ Only a few possible mechanisms explaining ipsilateral chorea were suggested. Vincent FM reported a case of unilateral chorea due to ipsilateral subdural hematoma. ${ }^{8}$ He postulated that midline shift or compression by hematoma lead to structural abnormality or dysfunction of contralateral cerebral hemisphere, which in turn caused ipsilateral chorea. Krauss et al. ${ }^{9}$ demonstrated two cases of hemichorea/hemiballism with contralateral hemiparesis caused by ipsilateral basal ganglionic lesions. They concluded that the motor deficit contralateral to the STN lesion triggers the appearance of the ipsilateral hyperkinesias due to bilateral motor control of STN. The last explanation suggested as mechanism of ipsilateral chorea was the releasing effect of ipsilateral lesion to the preexisting contralateral lesion. ${ }^{10}$ Dierssen et al. ${ }^{10}$ suggested that, from the cases of hemiballism after the second lesioning on thalamus, the effects of preexisting contralateral lesions were activated by releasing of ipsilateral lesions. These patients had previous contralateral stereotaxic thalamic lesion and abnormal movements occurred after production of the lesion in the other hemisphere. In the present case, no mass effect nor 
lesion on STN were found. Despite the preexisting left caudate nucleus lesion, newly developed infarction revealed by diffusion weighted images was limited to right ACA territory. Therefore we can only speculate the possible mechanism of the generalized chorea that acute ACA lesion made contralateral hemichorea and activated the contralateral caudate nucleus lesion which was dormant before the stroke.

Involuntary movements after ACA infarction had received little attention. Hemiparkinsonism and asterixis due to ACA infarction have been reported. ${ }^{7}$ However, generalized chorea associated with unilateral ACA infarction has rarely been reported. This case suggests that, although rare, unilateral ACA infarction can cause generalized chorea.

\section{REFERENCES}

1. Ghika-Schmid F, Ghika J, Regli F, Bogousslavsky J. Hyperkinetic movement disorders during and after acute stroke: the Lausanne Stroke Registry. J Neurol Sci 1997;146:109-116.
2. Alarcón F, Zijlmans JC, Dueñas G, Cevallos N. Post-stroke movement disorders: report of 56 patients. J Neurol Neurosurg Psychiatry 2004; 75:1568-1574.

3. Janavs JL, Aminoff MJ. Dystonia and chorea in acquired systemic disorders. J Neurol Neurosurg Psychiatry 1998;65:436-445.

4. Burnett L, Jankovic J. Chorea and ballism. Curr Opin Neurol Neurosurg 1992;5:308-313.

5. Yeterian EH, Van Hoesen GW. Cortico-striate projections in the rhesus monkey: the organization of certain cortico-caudate connections. Brain Res 1978;139:43-63.

6. Jurgens U. The efferent and afferent connections of the supplementary motor area. Brain Res 1984;300:63-81.

7. Kim JS. Involuntary movements after anterior cerebral artery territory infarction. Stroke 2001;32:258-261.

8. Vincent FM. Chorea: a late complication of a subdural hematoma. Neurology 1980;30:335-336.

9. Krauss JK, Pohle T, Borremans JJ. Hemichorea and hemiballism associated with contralateral hemiparesis and ipsilateral basal ganglia lesions. Mov Disord 1999;14:497-501.

10. Dierssen G, Gioino GG, Cooper IS. Participation of ipsilateral hemisphere lesions in the pathology of hemichorea and hemiballismus. Neurology 1961;11:894-898. 